\title{
Distribution of polymorphisms rs12979860, rs8099917 and rs12980275 IL28B in patients with chronic hepatitis C
}

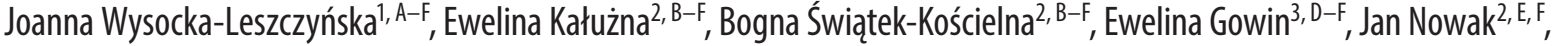

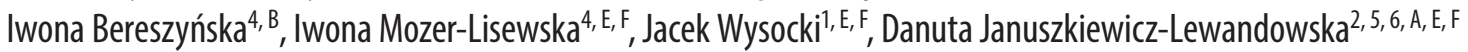 \\ ${ }^{1}$ Department of Health Prevention, Poznan University of Medical Sciences, Poland \\ 2 Department of Molecular Pathology, Institute of Human Genetics PASc, Poznań, Poland \\ ${ }^{3}$ Department of Family Medicine, Poznan University of Medical Sciences, Poland \\ ${ }^{4}$ Department and Clinic of Infectious Diseases, Poznan University of Medical Sciences, Poland \\ ${ }^{5}$ Clinic of Oncology, Hematology and Pediatric Transplantology, Poznan University of Medical Sciences, Poznań, Poland \\ ${ }^{6}$ Department of Medical Diagnostics, Poznań, Poland \\ A - research concept and design; B - collection and/or assembly of data; $C$ - data analysis and interpretation; \\ $D$ - writing the article; $E$ - critical revision of the article; $F$ - final approval of the article
}

Address for correspondence

Joanna Wysocka-Leszzzyńska

E-mail: leszczynskaj@interia.pl

Funding sources

None declared

Conflict of interest

None declared

Received on September 24, 2015

Reviewed on November 17, 2015

Accepted on October 19, 2016

\begin{abstract}
Background. The prognosis concerning the treatment of patients with chronic hepatitis C (CHC) is closely related to the genotype of the virus as well as to the factors dependent on the patient. It was proved that polymorphisms of the gene encoding interleukin 28B (IL28B) are associated with sustained viral response, which in the case of profitable variants of /L28B polymorphisms may reach up to $87 \%$ of the patients.
\end{abstract}

Objectives. The aim of the study is to determine the prevalence of alleles and distribution of IL28B polymorphisms genotypes in the examined group of patients with CHC in Wielkopolska Province.

Material and methods. A total of 710 people with diagnosed hepatitis C virus were examined in order to determine the distribution of polymorphisms of gene IL28B rs12979860, rs8099917 and rs12980275. The polymorphisms were evaluated by sequencing of PCR products.

Results. The most often noted profitable variant was genotype TT for polymorphism rs8099917 present in $43.5 \%$ of the patients, next was AA rs 12980275 in 22.5\%. The rarest was the profitable variant CC of the polymorphism rs 12979860 present in $17.5 \%$ of the patients. An occurrence of at least 2 IL28B polymorphisms in the preferred variants (homozygote CC, TT, AA) was found in 239 out of 710 (34\%) patients, among which 117 patients had favorable genotypes for all 3 examined polymorphisms.

Conclusions. The SNP distribution of gene IL28 with fixed prognostic value in the population of patients with chronic hepatitis C is different from the general population, and shows the need to evaluate polymorphisms prior to treatment.

Key words: HCV, hepatitis C, treatment

DOI

$10.17219 /$ acem $/ 66076$

Copyright

Copyright by Author(s)

This is an article distributed under the terms of the

Creative Commons Attribution Non-Commercial License

(http://creativecommons.org/licenses/by-nc-nd/4.0/) 


\section{Introduction}

Hepatitis $\mathrm{C}$ virus ( $\mathrm{HCV}$ ) is one of the largest epidemiological concerns in the world. It is estimated that nearly 170 million people are infected with that virus, which constitutes $2.2-3 \%$ of the world's population, among which as much as $70-80 \%$ of infected people remains chronically ill. The problem of effective treatment is associated i.a. with an occurrence of $7 \mathrm{HCV}$ genotypes, and over 100 of its subtypes. In the scale of Poland, this problem concerns about $1.5 \%$ of the population, and the predominant is type 1 infection (approx. $75 \%$ of patients), similarly as in other European countries (68-82\% of patients). ${ }^{1}$ Standard antiviral therapy is based on the application of pegylated interferon combined with ribavirin, but the achieved therapeutic response is still not satisfactory. Sustained viral response (SVR), defined as an absence of HCV RNA in the blood of patients 6 months after the 48-week treatment completion, is obtained by only $50 \%$ of people infected with type 1 who were subjected to the therapy. ${ }^{2}$ The effectiveness of the treatment is affected by a range of factors, depending on the patient and related to the viral genetic material. Numerous studies indicate that particular genotype of the gene encoding interleukin 28B (IL-28B) directly affects the treatment response in the infected people. The family of type III interferons, also known as interferons $\lambda$, consists of 3 types of proteins called: IFN- $\lambda 1$, IFN- $\lambda 2$, IFN- $\lambda 3$, or IL-29, IL$28 \mathrm{~A}$ and IL-28B, respectively, encoded by 3 genes located on chromosome $19 .{ }^{3}$ In contrast to type I, interferons encoded by the genes containing a single exon, the genes encoding IL-28A and IL-28B are composed of 6 exons,

Table 1. Sequences of starters and size of PCR reaction products for selected polymorphisms of interleukin $28 \mathrm{~B}$ gene

\begin{tabular}{|c|c|c|c|c|}
\hline Gene & Polymorphism & Starter & Starter sequence $\left(5^{\prime}-\ldots-3^{\prime}\right)$ & $\begin{array}{c}\text { Product } \\
\text { size }\end{array}$ \\
\hline \multirow{2}{*}{ IL28B } & \multirow{2}{*}{ rs12979860 } & $\mathrm{F}$ & GCGCTTATCGCATACGGCTA & \multirow{2}{*}{$189 \mathrm{bp}$} \\
\hline & & R & GGGGCGAGGGGCTTTGCT & \\
\hline \multirow{2}{*}{ IL28B } & \multirow{2}{*}{ rs8099917 } & F & AGTCTTGTATTTCACCTCCTGGAG & \multirow{2}{*}{$307 \mathrm{bp}$} \\
\hline & & R & GCACTAGCTCTTGTCATTTGCCT & \\
\hline \multirow{2}{*}{ IL28B } & \multirow{2}{*}{ rs12980275 } & F & GAAGGAAGTTCTGACACATGCT & \multirow{2}{*}{$280 \mathrm{bp}$} \\
\hline & & R & TGGTCCTAGTGGTGTTTGCT & \\
\hline
\end{tabular}

Table 2. Conditions of PCR reaction applied for the amplification of the samples for selected polymorphisms of interleukin 28 B gene

\begin{tabular}{|c|c|c|c|}
\hline \multicolumn{2}{|c|}{ Stage } & Temperature & Time \\
\hline \multicolumn{2}{|c|}{$\begin{array}{l}\text { Activation JumpStart }{ }^{\mathrm{TM}} \text { Taq } \\
\text { DNA Polymerase }\end{array}$} & $94^{\circ} \mathrm{C}$ & $3 \mathrm{~min}$ \\
\hline Denaturation & \multirow{3}{*}{$45 x$} & $94^{\circ} \mathrm{C}$ & $15 s$ \\
\hline $\begin{array}{l}\text { Starters } \\
\text { attachment }\end{array}$ & & $60^{\circ} \mathrm{C}^{1} / 57^{\circ} \mathrm{C}^{2} / 60^{\circ} \mathrm{C}^{3}$ & $30 \mathrm{~s}$ \\
\hline Synthesis & & $72^{\circ} \mathrm{C}$ & $30 \mathrm{~s}$ \\
\hline
\end{tabular}

${ }^{1}$ - rs12979860; ${ }^{2}$ - rs8099917; ${ }^{3}$ - rs12980275. and IL-29 contains 5 of them. ${ }^{4}$ Interferons $\lambda$ are subject to a direct induction as an effect of viral infections, like type I interferons.

The aim of this study is to determine the distribution of interleukin 28B polymorphisms in the group of patients from the area of Wielkopolska Province and to compare these results to the literature data concerning the distribution of these polymorphisms in patients from other countries and selected regions of Poland.

\section{Material and methods}

The study included the group of 710 patients (330 women and 380 men) staying under the care of the Clinic of Infectious Diseases of the Jozef Strus Municipal Hospital in Poznań (Poland) due to chronic hepatitis. The presence of the genetic material of hepatitis $C$ virus was confirmed in all the patients, and the genotype of the virus was identified in some of the patients.

In case of 217 patients, the starting material for examination were oral mucosa epithelial cells. Invisorb ${ }^{\circledR}$ Spin Tissue Mini Kit (Stratec molecular) was applied for the isolation of genomic DNA in these samples. The procedure of isolation was performed according to the manufacturer's instructions. The starting material for the other 493 samples was peripheral blood collected on EDTA and centrifuged at density gradient Histopaque ${ }^{\circledR}-1077$ (SigmaAldrich, St. Louis, USA) in order to obtain a mononuclear cells fraction PBMCs (peripheral blood mononuclear cells). Genomic DNA was isolated from $1 \times 10^{6}$ PBMCs suspended in phosphate buffered saline (PBS) (to $200 \mu \mathrm{L}$ ) using QIAamp ${ }^{\circledR}$ DNA Mini and Blood Mini Kit (Qiagen, Hilden, Germany). The procedure of isolation was performed according to the manufacturer's instructions.

The samples were then amplified using the PCR reaction, obtaining the products with the sizes presented in Table 1.

PCR reaction was performed in a total sample volume of $25 \mu \mathrm{L}$. The sample contained reaction buffer with $15 \mathrm{mM} \mathrm{MgCl} 2$, dNTPs at an initial concentration of $10 \mathrm{mM}$, JumpStart Taq ${ }^{\mathrm{TM}}$ DNA Polymerase (Sigma-Aldrich, St. Louis, USA) at a concentration of $2.5 \mathrm{IU} / \mu \mathrm{L}, \mathrm{F}$ and $\mathrm{R}$ primers at concentrations of $20 \mu \mathrm{M}$, matrix DNA, and water. In addition, $25 \mathrm{mM} \mathrm{MgCl}_{2}$ solution was used in the reaction mixture for rs8099917 and rs12980275 polymorphisms.

The conditions of PCR reaction are presented in Table 2.

The resulting products were purified using NucleoSpin ${ }^{\circledR}$ Gel and PCR Clean-up (MachereyNagel, Duren, Germany) set in accordance with the manufacturer's instructions, and then sequenced using the method of Sanger. 
The prevalence of genotypes in the examined group was compared with the expected prevalence resulting from the Hardy-Weinberg equilibrium, using a statistical calculator provided by Tufts University (USA). The analysis of linkage disequilibrium (LD) was performed using the HaploView 4.2 software (Broad Institute, Harvard, USA).

\section{Results}

The analysis of rs12979860 polymorphism showed a profitable genotype CC in 124 patients (17.5\%), genotype CT in 443 patients (62.4\% of the examined group), and genotype TT in 143 patients (20.1\%). In the case of rs8099917 polymorphism, the presence of profitable genotype TT was found in 309 patients (43.5\%), genotype GT in 346 patients (48.9\% of the examined group), and genotype GG in 54 patients (7.6\%). The third of the examined polymorphisms ( $\mathrm{rs} 12980275)$ in a preferred variant AA was noted in 158 patients (22.3\%), AG in 426 patients representing $60 \%$ of the examined group, and genotype GG was found in 126 patients (17.7\%). Graphical comparison of the results is presented in Fig. 1.

The prevalence of alleles and the distribution of examined polymorphisms genotypes are presented in Table 3. The relationship of the occurrence of the profitable polymorphisms is shown in Fig. 2.

The presence of at least 2 polymorphisms IL28B in profitable variants (CC, TT, AA) was found in 239 patients $(33.7 \%)$, and the occurrence of profitable homozy-

Table 3. Prevalence of alleles and distribution of gene IL28B polymorphisms (rs12979860, rs8099917 and rs12980275) in the examined group of CHC patients

\begin{tabular}{|c|c|c|c|}
\hline Polymorphism & Change & Allele/genotypes & $\mathrm{n}(\%)$ \\
\hline \multirow{6}{*}{ rs12979860 } & \multirow{6}{*}{$\mathrm{C} / \mathrm{T}$} & C & $691(48.7)$ \\
\hline & & $\mathrm{T}$ & $729(51.3)$ \\
\hline & & $\mathrm{CC}$ & $124(17.5)$ \\
\hline & & $C T$ & $443(62.4)$ \\
\hline & & $\mathrm{TT}$ & $143(20.1)$ \\
\hline & & $\mathrm{CC} / \mathrm{CT}+\mathrm{TT}$ & $124(17.5) / 586(82.5)$ \\
\hline \multirow{6}{*}{ rs8099917 } & \multirow{6}{*}{$\mathrm{T} / \mathrm{G}$} & $\mathrm{T}$ & $965(68.0)$ \\
\hline & & G & $455(32.0)$ \\
\hline & & $\mathrm{TT}$ & $309(43.5)$ \\
\hline & & $\mathrm{TG}$ & $347(48.9)$ \\
\hline & & GG & $54(7.6)$ \\
\hline & & $\mathrm{TT} / \mathrm{TG}+\mathrm{GG}$ & 309 (43.5) / 401 (56.5) \\
\hline \multirow{6}{*}{ rs12980275 } & \multirow{6}{*}{$A / G$} & A & $742(52.3)$ \\
\hline & & G & $678(47.7)$ \\
\hline & & AA & $158(22.3)$ \\
\hline & & $A G$ & $426(60.0)$ \\
\hline & & GG & $126(17.7)$ \\
\hline & & $A A / A A+A G$ & $158(22.3) / 552(77.7)$ \\
\hline
\end{tabular}

gotes for all 3 examined polymorphisms was noted in 117 patients among them (49 women and 68 men), while beneficial homozygotes for the first 2 of the examined polymorphisms was observed in 122 patients (52 women and 70 men). These results are presented in Fig. 2.

It was found that the distribution of genotypes for all 3 examined polymorphisms did not comply with the distribution expected according to the Hardy-Weinberg law $(\mathrm{p}<0.05)$.

The linkage disequilibrium was moderate between rs12979860 and rs8099917 ( $\mathrm{r} 2=0.43$ ) as well as between rs8099917 and rs12980275 (r2=0.48). The rs12979860SNP was in strong LD with rs12980275 (r2 = 0.78). The D' values for the rs12979860, rs8099917 and rs12980275 was $>0.95$. D' values for rs12979860 and rs8099917, rs8099917 and rs12980275, rs12979860 and rs12980275 were: 0.98; 0.96 and 0.95 , respectively.

The most common haplotypes of the examined polymorphisms were CTA, TGG, and TTG (47.3\%, 31.5\% and $15.1 \%$, respectively). Other haplotypes (TTA and CTG) occurred at a frequency less than $5 \%(4.4 \%$ and $1.1 \%$, respectively).

\section{Discussion}

Distribution of gene IL28 polymorphisms obtained in the examined group is different from the general population. It is estimated that among Caucasian patients the incidence of CC variant of rs12979860 polymorphism is about $40 \%$. Global research indicates that the highest prevalence of genotype CC is observed in Asians (over 90\%); it is lower in Europeans, Americans and Latinos, and the lowest in Africans. ${ }^{5}$

Fig. 1. Graphical comparison of the allele distribution
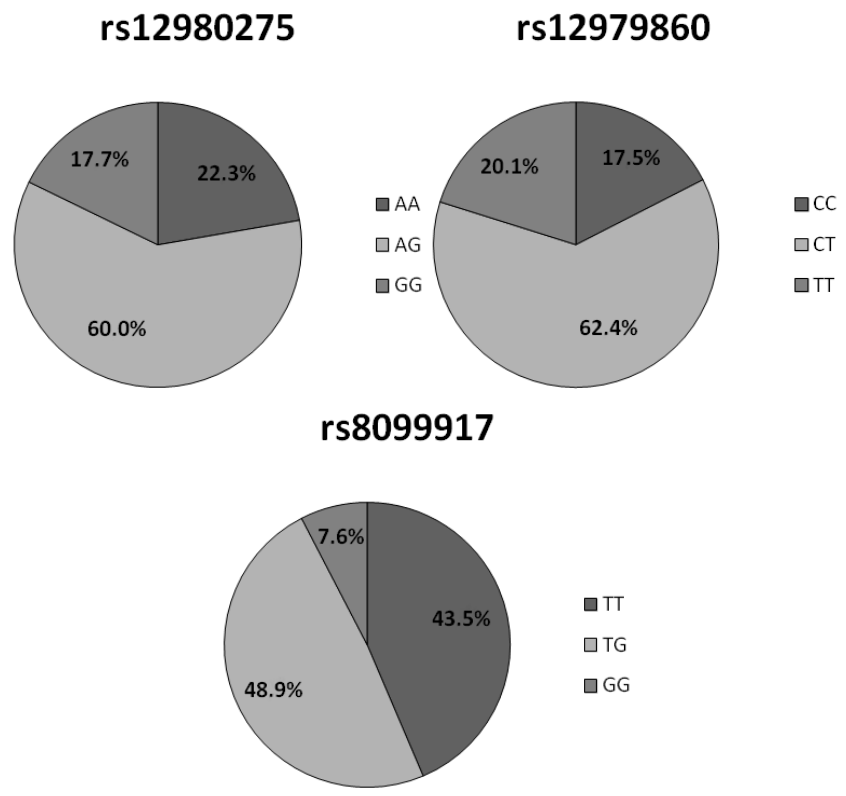
So far, it was proved that rs12979860 polymorphism $(\mathrm{C} / \mathrm{T})$ in homozygous $\mathrm{CC}$ variant affects the higher frequency of sustained viral response compared to genotype TT, and increases the chance of spontaneous elimination of HCV infection. ${ }^{6}$ Thompson determined in his study that an early viral response (EVR) is obtained by as much as $87 \%$ of the patients with genotype CC, compared to the $38 \%$ and $28 \%$ with genotypes CT and TT, respectively. ${ }^{7}$ Further research on the effectiveness of antiviral therapy in the patients with chronic hepatitis $\mathrm{C}$ led to the discovery that the profitable course of treatment in the case of polymorphism rs 8099917 (T/G) is observed in the patients with genotype TT, while for polymorphism rs12980275 (A/G ) these observations apply to the patients with genotype AA. ${ }^{8}$

Profitable variants may determine the phenomenon of spontaneous $\mathrm{HCV}$ elimination, but do not provide the resistance to infection upon exposure. It was demonstrated, based on observations of the group of patients after the exposure to hepatitis $\mathrm{C}$ virus, that the presence of variant CC does not protect against an acute infection, whereas homozygocity for KIR2DL3:HLA-C1 provides such protection. ${ }^{9}$

In the case of polymorphism rs12979860, the predominant observed genotype in the examined group was variant CT (62.5\% of the examined group), which also dominates in the groups of patients examined in Bydgoszcz and Białystok (56.0\% and 60\%, respectively). ${ }^{10,11}$ Concurrently, the obtained percentage of patients with advantageous variant CC occurs at a similar level of about $20 \%$. This ratio is slightly different in the case of patients examined in Kraków - the percentage of people with variants CT and CC was at a similar level, and amounted to $47 \%$ and $42 \%$ of the examined group, respectively. ${ }^{12}$ At the same time, a respectively lower percentage of people with genotype TT (11.0\%) was found in this group compared to the groups examined in Poznań, Bydgoszcz and Białystok (20.0\%, 16.0\% and 20.0\%, respectively). Analyzing the results obtained in Italy, Germany or Hungary, it can be observed that the prevalence of people with genotype CT remains at a very similar level of about $50 \%$, while with genotype TT constitutes more than $10 \%$ of the examined patients. ${ }^{13-15}$ This is explained by the hypothesis that the variant TT may have a protective effect against acute infection occurrence. ${ }^{8}$

Comparison of the frequency of particular variants in different research studies reveals significant discrepancies. This depends on the selection of the examined group: ethnicity of the patients, accompanying HIV or HBV infection, disease severity - patients with newly diagnosed infection by screening, or patients with chronic infection qualified to the treatment, patients not responding to standard treatment.

The detection of unprofitable variant being the factor worsening the prognostics concerning the successful treatment in the patient suffering from chronic hepati- tis $\mathrm{C}$ may be a prerequisite for the use of triple therapy. Triple therapy was introduced in Poland in 2013, which involved the addition of the $3^{\text {rd }}$ antiviral preparation to the existing treatment - a protease inhibitor (telaprevir or boceprevir). Currently, the availability of this therapy in Poland is limited, and the criteria of eligibility include i.a. the patients suffering from chronic hepatitis $\mathrm{C}$ infected with genotype $1 \mathrm{HCV}$. In the case of the patients not treated previously, triple therapy costs are reimbursed by the National Health Fund only to the patients who suffer from progressive liver fibrosis (grade 2 upwards on the Scheuer's scale) and who have been diagnosed with the genotype TT for polymorphism rs12979860 IL28B. The patients who did not respond to previous dual therapy may be considered for the treatment in triple therapy program only in the case of progressive liver fibrosis (grade 2 upwards on the Scheuer's scale). For this reason, the examination of genotype IL-28B becomes extremely important in Poland, since it directly affects the decision to finance the treatment by the National Health Fund.

The prognosis concerning the effective treatment was also determined for the other 2 polymorphisms of gene IL28B - rs8099917 and rs12980275, but they have a weaker predictive value concerning the response to therapy. ${ }^{8}$ Among the examined patients from the Wielkopolska Province, the occurrence of at least 1 of the preferred variants was noted in 591 cases. The most frequent advantageous variant was TT rs 8099917 reported in 43.5\% of the patients; almost all of these patients were the carriers of profitable variant CC rs12979860 (except 2 patients). The occurrence of at least 2 preferred variants was noted in $34 \%$ of the patients, and $16 \%$ of the examined patients were the carriers of 3 preferred variants. It was found that in the case of those patients who did not have the preferred variant CC rs12979860, 187 of the patients

Fig. 2. Graphical comparison of the results

rs12979860

rs8099917
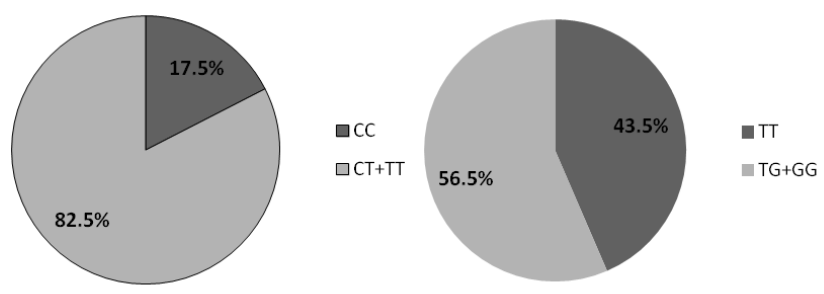

rs12980275

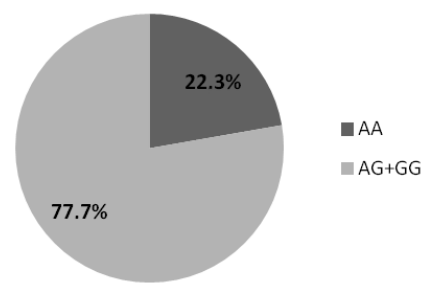


(26.3\%) had the preferred variant TT rs8099917, and 36 patients (5\%) had the preferred variant of both polymorphisms (TT and AA rs8099917 and rs12980275).

It may be concluded on that basis that more than $1 / 3$ of the examined patients are those for whom the application of treatment with pegylated interferon in combination with ribavirin is associated with good prognostics concerning the cure. No additional benefits concerning the concurrent presence of several preferred variants of various SNPs of gene IL28 have been demonstrated so far. ${ }^{8}$ In the case of the preferred variants of other genes polymorphisms, e.g., KIR2DL3: HLA-C1 and CC LL28, their variants observed concurrently do not give any additional profits. Both mechanisms operate independently and do not act synergistically.

The analysis of the coexistence of the preferred variants of particular polymorphisms demonstrated that almost all the patients with profitable polymorphism rs12979860 had also a beneficial variant of polymorphism rs8099917. Variant TT rs8099917 was the most common preferred polymorphism in the examined population; hence, it can be considered that its determination will allow us to indicate the largest group of patients with assumed positive effects of interferon treatment. From the economic point of view, it is reasonable to determine the polymorphism rs8099917 only in the patients who did not show a preferred variant of rs12979860.

The distribution of SNP gene IL28 with fixed prognostic value in the population of patients with chronic hepatitis $\mathrm{C}$ is different from the general population, and shows the need of an evaluation of polymorphisms prior to the treatment. Due to the lack of additional effect of several preferred variants coexistence, the need to determine all 3 polymorphisms in each patient, or specify whether such examinations should be carried out only in case of an absence of preferred polymorphism variant of the highest prognostic value, should be explained. Knowledge of the prevalence of the preferred variants of particular polymorphisms in the population of patients with chronic hepatitis $C$ will enable selecting the most efficient procedure allowing the evaluation of prognosis.

\section{References}

1. Tyczyno M. HCV genotypes among patients diagnosed in Bydgoszcz. Med Sci Rev Hepatol. 2007;7:77-79.

2. Manns MP, McHutchison JG, Gordon SC, et al. Peginterferon alfa-2b plus ribavirin compared with interferon alfa- $2 \mathrm{~b}$ plus ribavirin for initial treatment of chronic hepatitis C: A randomized trial. Lancet. 2001;358:958-965.

3. Domagalski K, Tretyn A, Pawłowska M, Szczepanek J, Halota W. Action of type III IFNs and their roles in immune responses. Postepy Hig Med Dosw. 2010;64:522-533.

4. Kotenko S, Gallagher G, Baurin VV, et al. IFN- $\lambda$ s mediate antiviral protection through a distinct class II cytokine receptor complex. Nature Immunology. 2003;4:69-77.

5. Ge D, Fellay J, Thompson Aj, et al. Genetic variation in IL28B predicts hepatitis $C$ treatment-induced viral clearance. Nature. 2009;461:399-401.
6. Thomas DL, Thio CL, Martin MP, et al. Genetic variation in IL28B and spontaneous clearance of hepatitis C virus. Nature. 2009;461:798-801.

7. Thompson AJ, Muir AJ, Sulkowski MS, et al. Interleukin 28B polymorphism improves viral kinetics and is the strongest pre-treatment predictor of sustained virologic response in genotype 1 hepatitis C virus. Gastroenterology. 2010;139:120-129.

8. Aparicio E, Parera M, Franco S, et al. IL28B SNP rs8099917 is strongly associated with pegylated interferon- $a$ and ribavirin therapy treatment failure in HCV/HIV-1 coinfected patients. PLoS One. 2010;29:e13771. doi: 10.1371/journal.pone.0013771.

9. Knapp S, Warshow U, Ho KMA, et al. A polymorphism in IL28B distinguishes exposed, uninfected individuals from spontaneous resolvers of HCV infection. Gastroenterology. 2011;141:320-325.

10. Domagalski K, Pawłowska M, Tretyn A, et al. Association of IL28B with the response to peginterferon plus ribavirin combined in Polish patients infected with HCV genotype 1 and 4. Hepat Mon. 2013;13:e13678. doi: 10.5812/hepatmon.13678.

11. Kozłowski P, Pogorzelska J, Łapiński TW, et al. The occurrence of HCV genotypes and single polynucleotide polymorphisms of rs12979860 among HCV infected patients in northeastern Poland. Przegl Epidemiol. 2012;66:25-31.

12. Kaczor MP, Seczyńska M, Szczeklik W, Sanak M. IL28B polymorphism (rs12979860) associated with clearance of HCV infection in Poland: Systematic review of its prevalence in chronic hepatitis $C$ patients and general population frequency. Pharmacological Reports. 2015;67:260-266.

13. Fabris C, Falleti E, Cussigh A, et al. IL-28B rs12979860 C/T allele distribution in patients with liver cirrhosis: Role in the course of chronic viral hepatitis and the development of HCC. J Hepatol. 2011;54: 716-722.

14. Lutz P, Wasmuth JC, Nischalke HD, et al. Progression of liver fibrosis in HIV/HCV genotype 1 co-infected patients is related to the T allele of the rs12979860 polymorphism of the IL28B gene. Eur J Med Res. 2011;16:335-341.

15. Par A, Par G, Tornai I, et al. IL28B and IL10R -1087 polymorphisms are protective for chronic genotype $1 \mathrm{HCV}$ infection and predictors of response to interferon-based therapy in an East-Central European cohort. BMC Research Notes. 2014;7:12. 
